IJMS 2019 vol. 6 (1): 1 - 6

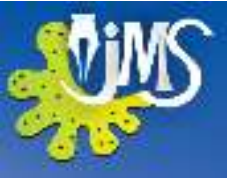

International Journal of Multidisciplinary Studies (IJMS)

Volume 6, Issue 1, 2019

DOI: http://doi.org/10.4038/ijms.v6il.86

\title{
Development of sodium-ion rechargeable battery using sodium cobalt phosphate cathode
}

\author{
Wijesinghe H.D.W.M.A.M. ${ }^{1}$, Manathunga C.H. ${ }^{1}{ }^{*}$ and PereraV.P.S. ${ }^{2}$ \\ ${ }^{1}$ Department of Physics, Faculty of Applied Sciences, University of Sri Jayewardenepura, Nugegoda, \\ Sri Lanka \\ ${ }^{2}$ Department of Physics, Faculty of Natural Sciences, Open University of Sri Lanka, Nawala, Nugegoda, \\ Sri Lanka
}

\begin{abstract}
Lithium-ion batteries are the most popular kind of rechargeable batteries accommodate in portable electronic devices up to date. As the Lithium deposits are depleting the cost of Lithium-ion batteries is increasing. Sodium- ion batteries can be introduced as an alternative technology which can replace expensive Lithium-ion batteries. Sodium sources are highly abundant and therefore Sodium-ion batteries could be made cheaper than Lithium-ion batteries. A number of cathode materials which were accommodated in Lithium-ion batteries have also been tested as cathode materials for Sodium-ion batteries. This research was based on a Sodium-ion battery which cathode was prepared using Sodium cobalt phosphate. The cathode material was prepared using a simple solid-state reaction between Cobalt (II) oxide and Sodium phosphate. The prepared material was characterized using powder XRD. Chargedischarge cycles, cyclic voltammetry analyzing, impedance curve matching to obtain equivalent circuit was used in order to analyze the performance of the prepared cathode in the battery. The discharge capacity of the cathode was calculated as $9.58 \mathrm{~mA} \mathrm{~h} \mathrm{~g}^{-1}$. The cyclic voltammetry curve has shown that an oxidation and reduction processes involved in the battery cycle but the battery cycle was not completely reversible.
\end{abstract}

KEYWORDS: Rechargeable batteries, Sodium cobalt oxide, cyclic voltammetry, charge-discharge cycles. 


\section{INTRODUCTION}

As the development of portable electronic devices increased rapidly the rechargeable battery technology developed vastly.. Lithium-ion (LIB) battery was the pinnacle of the rechargeable batteries up to this date (Nitta et al., 2015).

However, limited availability of Lithium deposits makes the cost of these batteries increasing and an alternative solution must be brought to light. In this case, Sodium-ion batteries (SIB) are among the perspective candidates to replace LIBs in the near future (Hwang et al., 2017). High abundance of Sodium deposits is a huge advantage in cost cutting through the process of presenting SIBs to the market.

The primary objective of the present study was to prepare a cathode for Sodium-ion batteries using Sodium cobalt phosphate and explore its capacity and recharge ability. The preparation of the active material was driven through a solid-state reaction between cobalt oxide and sodium phosphate.

\section{Background for the study}

The lithium-ion battery was a radical invention that made the dream of a long living rechargeable battery a reality. Lithium-ion batteries are originally developed as a high energy power source for electronic devices limited to $100 \mathrm{~W}$ h (Nitta et al., 2015). They were typically limited as a single battery pack. Cobalt/nickel-based materials with layered structures are typically used as positive electrodes for high energy but small sized batteries (De Silva et al., 2014). A number of cathode material groups were tested and employed for Lithium-ion batteries. Intercalation cathode materials such as metal chalcogenides, transition metal oxides, Polyanion compounds are among them (Nitta et al., 2015).

The major disadvantage of Lithium-ion batteries is the high cost. The reason behind this is that the Lithium deposits are rare and they are getting depleted. Sodium ion batteries have been attracting more and more researchers meanwhile due to the fact that the high abundance of sodium reduces the cost (Zhao et al., 2016). Although research interest about Sodium-ion batteries are

relatively younger than the other battery technologies, it has a great potential because of its low cost (De Silva et al., 2014).

Electrode performance of layered sodium transition metal oxides, which was originally studied as positive electrode materials for Sodium batteries in the 1980s, has been significantly improved by the latest battery technology developed in the Lithium-ion battery system during the past three decades (Kubota et al., 2014). Not only oxides but also phosphates of transition metals are considered as high energy storing cathode materials for sodium ion batteries. Phosphate framework materials consist of a number of varieties that are versatile and flexible structure and with good electrochemical performance. These phosphate varieties include phosphates, pyrophosphates, mixed anions, and diverse optional redox centers (Fe, V, Mn, Ni, Co). From the viewpoint of electrochemistry, most of the reactions are attributed to phase transition mechanism, and some belong to 
solid solution, surface or interface charging (Fang et al., 2017).

Cobalt is a transition metal which has two main oxidation states (+II and +III). Sodium cobalt oxides were employed as a cathode material since the early $1980 \mathrm{~s}$ (Su et al., 2016) for rechargeable batteries. Most of the previously known methods in synthesizing were based on solution combustion techniques (Gond et al., 2017) other than solid-state reactions. But there is no much work done on Sodium Cobalt phosphate as the cathode material of sodium ion batteries.

\section{MATERIALS AND METHODS}

\section{Fabrication of Cathode Material}

Cathode material was synthesized using a simple method. As the first step Cobalt (II) oxide and Sodium phosphate was taken in 1:1 mole ratio and grinded using a mortar and pestle until a homogeneous powder was formed. This mixture was loaded into a crucible and calcinated at around 800 0C in a muffle furnace for one-hour. Then the sample was grinded again and calcinated for another half an hour at the same temperature. This process was carried out for three times.

\section{Material Characterisation}

X-ray diffraction (XRD) characterization was performed on the resulting powder with Regaku Ultima VI, X-ray Diffractometer using $\mathrm{Cu} \mathrm{K} \alpha(\lambda=$

$1542 \AA$ ) radiation to analyze the structure of the sample.

\section{Cell Fabrication}

The active material, activated carbon and Polyvinylidene Fluoride (PVDF) were mixed in the ratios of 18:1:1 mass ratio. The prepared slurry was pasted on an Aluminium foil and dried on a hot plate at about 100 0C in order to prepare the cathode using the doctor blade method (Alahakoon et al., 2017). Metallic Sodium pressed onto Copper plate was used as the anode. The electrolyte was prepared using $1 \mathrm{~mol} \mathrm{dm}-3, \mathrm{NaClO} 4$ (sodium perchlorate). Dissolved in propylene carbonate and cellulose separator in between the electrodes was soaked with the electrolyte. Cell was fabricated in an Argon gas filled glove box.

\section{Cell Testing}

Charge discharge, cyclic voltametric cycles and impedance analyzing measurements were monitored and taken using the Autolab FRA 32 Galvanostat/Potentiostat. Here 0.5 $\mathrm{mA}$ current was used to charge as well as to discharge the cell. Cyclic voltammetric analysis was done using $0.05 \mathrm{~V} \mathrm{~s}-1$ scan rate.

Impedance spectroscopic measurements were obtained by using Autolab FRA 32 in the frequency range of $1.0 \mathrm{MHz}$ to 10.0 $\mathrm{mHz}$. Obtained data was used to find the impedance of cathode material and to construct equivalent circuit matching with the impedance data. These operations were performed using Nova 2.1 software.

\section{RESULTS \& DISCUSSION}

The peaks in the XRD pattern (Figure 1) matches with the reported literature of NaCoPO4 (Gond et al., 2017, Senthilkumar 
et al., 2014). Therefore, the solid-state reaction of cobalt oxide and sodium phosphate supposed to produce the active material. The $d$ values (in angstrom) and $2 \theta$ (in degrees.) values related to Peaks were [1] $\mathrm{d}=4.275 / 2 \theta=20.76$ [2] $\mathrm{d}=2.862 / 2 \theta=31.226$

[3] $\mathrm{d}=2.6145 / 2 \theta=34.269$ $\mathrm{d}=2.4405 / 2 \theta=36.80 \quad$ [5] $\mathrm{d}=1.5573 / 2 \theta=59.29$

[6] $\mathrm{d}=1.4647 / 2 \theta=63.445$

$\mathrm{d}=1.4299 / 2 \theta=65.19[8] \mathrm{d}=1.3956 / 2 \theta=67.00$ [9] $\mathrm{d}=1.284 / 2 \theta=73.7$.

Voltage variation with time during charging the cell is shown in figure 2. Cell charging was done with a current of $0.5 \mathrm{~mA}$. The charging cycle reaches about $1.9 \mathrm{~V}$ initially and then it stabilized at about $1.6 \mathrm{~V}$ with the time. A small drop in the voltage of the battery could be seen after that, however, the voltage did not drop further below $1.5 \mathrm{~V}$ during the charging cycle.

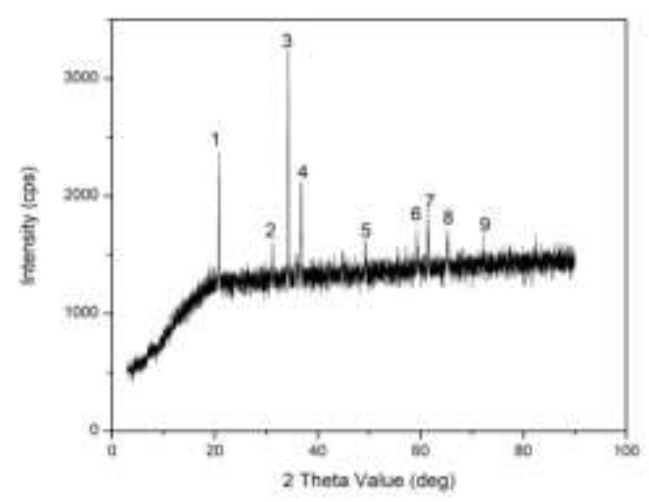

Figure 1: Obtained powder XRD pattern for the prepared cathode material

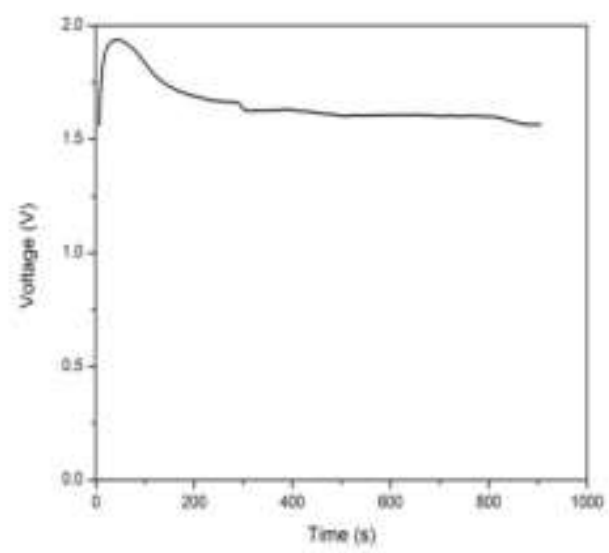

Figure 2: The typical charging cycle of cell

The typical discharging curve has shown a steep fall from $1.6 \mathrm{~V}$ to about $0.1 \mathrm{~V}$ within few seconds (Figure 3). After that, it maintained that particular voltage for about 4334 seconds before it is fully discharged to zero volts. Discharging curve pattern hints about Oxidation/reduction reaction. The discharging capacity was calculated to be as $9.58 \mathrm{~mA}$ h g-1.

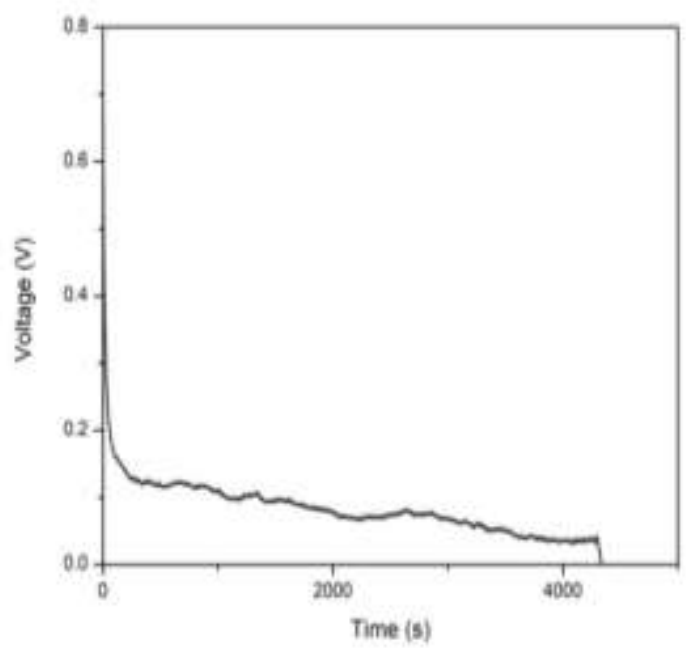

Figure 3: The typical discharging cycle of cell 
Oxidation and reduction peaks depicted at $1.75 \mathrm{~V}$ and $0.5 \mathrm{~V}$ respectively in the cyclic voltametric measurements of the cell (Figure 4). The reduction peak height is less than the oxidation peak which indicates that the charge cycle is irreversible.

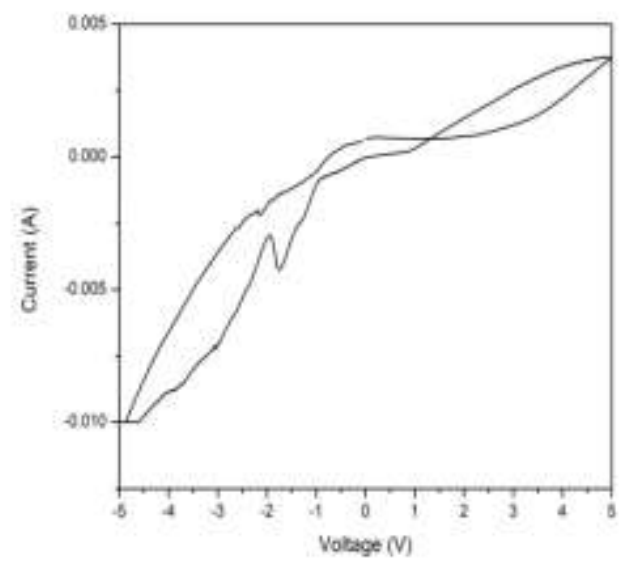

Figure 4: The cyclic voltammetry graph of the prepared cell.

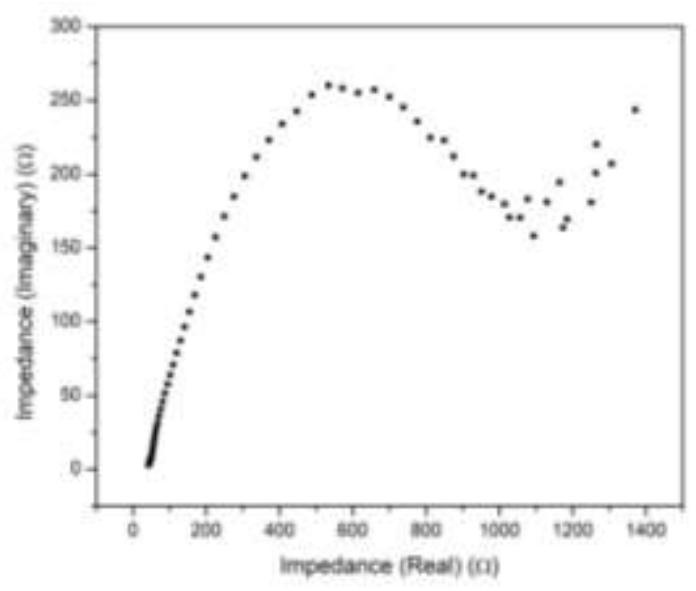

Figure 5: Nyquist plot of the battery

Figure 5 shows the Nyquist plot of the fabricated battery drawn using the Impedance spectroscopic measurements. The equivalent circuit matching with the impedance data is presented in the insertion. These data provide the presence of two interfaces probably the interface between the cathode and anode with the electrolyte. The smaller semicircle at the high-frequency end represents the interface of the electrolyte with sodium anode and a larger semicircle at low-frequency end represents the interface between the electrolyte and the cathode. The Warburg impedance indicated the ionic

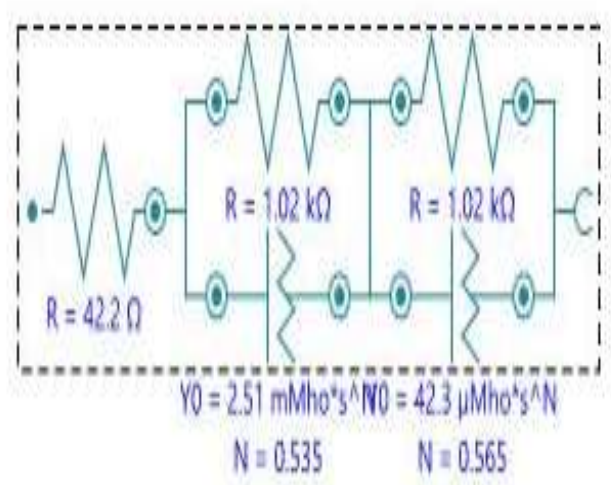

conductivity of the electrolyte.

Figure 6: The equitant circuit for the battery

The equivalent circuit of the cell is given in Figure 6. It is indicating the charge transfer resistances and impedance of constant phase elements.

\section{CONCLUSION/FURTHER WORK}

Sodium cobalt phosphate was successfully synthesized using Cobalt (II) oxide and Sodium phosphate in a solid-state reaction. The XRD data confirmed the synthesis of active material used to fabricate the cell. The calculated discharge capacity of the cell was $9.58 \mathrm{~mA} \mathrm{~h} \mathrm{g-1.} \mathrm{It} \mathrm{is} \mathrm{evident} \mathrm{from} \mathrm{the} \mathrm{cyclic}$ Volta monograph that the charge cycle is not reversible. Therefore, it was difficult to 
fabricate Na-ion rechargeable cell with a very high capacity. It is reported in the literature that the activity and stability of disordered Na2CoP2O7 higher than NaCoPO4 (Gond et al., 2017). Therefore, future work has to be directed towards to improve the cyclability and capacity of these batteries.

\section{ACKNOWLEDGMENT}

The authors would like to show their gratitude to the Research Council of the University of Sri Jayewardenepura for providing financial support for this research (ASP/01/RE/SCI/2016/24). Further authors are thankful to the Centre for Advanced Material Research, University of Sri Jayewardenepura and the Open University of Sri Lanka for providing laboratory facilities.

\section{REFERENCES}

Alahakoon, T. N., Perera, V. P. S., Shantha, N. G. S. \& Manathunga, C. H. (2017). Fabrication of Sodium Ion Rechargeable Battery Using Earth Abundant Orthosilicates Proceeding . 15th Open University Research Sessions (OURS 2017)

De Silva, R. C. L., Jayaweera, M. T. V. P., Perera, V. P. S., Jayarathna, I. P. L. \& Rosa, S. R. D. (2014). Sodium nickel oxide nanoporous cathodes used for sodium-ion rechargeable batteries. Sri Lankan Journal of Physics,15,.pp 19-29.

Fang, Y., Zhang, J., Xiao, L., Ai, X., Cao, Y. \& Yang, H. (2017). Phosphate Framework Electrode Materials for Sodium Ion Batteries. Adv Sci (Weinh), 4, 1600392.
Gond, R., Sada, K., Senthilkumar, B. \& Barpanda, P. (2017). Bifunctional Electrocatalytic Behavior of Sodium Cobalt Phosphates in Alkaline Solution. ChemElectroChem, 5, pp. 153-158.

Hwang, J. Y., Myung, S. T. \& Sun, Y. K. (2017). Sodium-ion batteries: present and future. Chem Soc Rev, 46, pp. 3529-3614.

Kubota, K., Yokoh, K., Yabuuchi, N. \& Komaba, S.( 2014). Na2CoPO4F as a Highvoltage Electrode Material for Na-ion Batteries. Electrochemistry, 82, pp. 909-911.

Nitta, N., Wu, F., Lee, J. T. \& Yushin, G. (2015). Li-ion battery materials: present and future. Materials Today, 18, pp. 252-264.

Senthilkumar, B., Sankar, K. V., Vasylechko, L., Lee, Y.S. \& Selvan, R. K. (2014). Synthesis and electrochemical performances of maricite-NaMPO4 ( $\mathrm{M}=$ $\mathrm{Ni}, \mathrm{Co}, \mathrm{Mn})$ electrodes for hybrid supercapacitors. RSC Adv., 4, pp.5319253200

Su, H., Jaffer, S. \& Yu, H. (2016). Transition metal oxides for sodium-ion batteries. EnergyStorage Materials,5, pp. 116-131.

Zhao, W., Zhong, G., Mcdonald, M. J., Gong, Z., Liu, R., Bai, J., Yang, C., LI, S., Zhao, W., Wang, H., FU, R., Jiang, Z. \& Yang, Y.(2016). Cu 3 (PO 4 ) 2 /C composite as a high-capacity cathode material for rechargeable Na-ion batteries. Nano Energy, 27, pp.420-429. 